\title{
Detection of Coronary Plaque by Computed Tomography With a Novel Plaque Analysis System, 'Plaque Map', and Comparison With Intravascular Ultrasound and Angioscopy
}

\author{
Sei Komatsu, MD; Atsushi Hirayama, MD; Yosuke Omori, MD; Yasunori Ueda, MD; \\ Isamu Mizote, MD; Yasuo Fujisawa*; Masayoshi Kiyomoto*; \\ Toshiaki Higashide*; Kazuhisa Kodama, MD
}

\begin{abstract}
Background Previous reports suggest that plaque may be characterized by the computed tomography (CT) number, but there is not a comprehensive method for evaluating the gray-scale CT image of the coronary artery obtained by multi-detector row CT (MDCT).

Methods and Results Forty-five patients with acute coronary syndrome (ACS) underwent MDCT either 3-4 weeks after the onset of acute myocardial infarction $(n=24)$ or within 1 week after percutaneous coronary intervention in patients with unstable angina (UA; $n=21)$. The cross-sections obtained at intervals of $5 \mathrm{~mm}$ were converted to numerical data and a 'plaque map' was drawn using the color-based isometric line method and bird's eye view. 'Plaque map' was compared with the findings of intravascular ultrasound (IVUS) and angioscopy. Of 662 slices of 78 vessels, soft, intermediate or calcified plaque was detected in 144, 134, and 84 slices, respectively. Compared with IVUS, the sensitivities were $92 \%, 87 \%$, and $89 \%$, respectively, and compared with angioscopy, sensitivity was $80 \%$ and specificity was $87 \%$.
\end{abstract}

Conclusions MDCT with the 'Plaque Map' system can noninvasively characterize plaque in patients with ACS. (Circ J 2005; 69: 72-77)

Key Words: Acute coronary syndrome; Angioscopy; Intravascular ultrasound; Multi-detector row computed tomography; Plaque

D isruption of vulnerable plaque may cause acute coronary syndrome $(\mathrm{ACS})^{1-4}$ and at present angioscopy ${ }^{5-9}$ and intravascular ultrasonography (IVUS) $)^{10-15}$ are the most powerful, though invasive, methods of precisely evaluating vulnerable plaque. An angioscopic study reported that vulnerable plaque development was observed in culprit as well as nonculprit coronary artery segments in patients with myocardial infarction, Yellow plaque detected by angioscopy is reportedly related to ACS 16,17 and the grade of yellow plaque predicts its vulnerability ${ }^{5-8}$ Hodgson et al reported that soft plaque was detected more often than calcified plaque by IVUS in an ACS patient $1^{10}$ Calcified plaque may be involved in cases of sudden death 18

The feasibility of multi-detector row computed tomography (MDCT) ${ }^{19-22}$ for coronary stenosis, 23,24 detection of soft plaque ${ }^{25}$ and measuring the vessel area by manual tracing 26 has been reported. MDCT has undergone development of the number of detectors required to obtain precise images ${ }^{27,28}$ Schroeder et al used the region of interest (ROI) to classify plaque by computed tomography (CT) number ${ }^{27}$ and very recently Achenbach et al reported a

(Received September 13, 2004; revised manuscript received October 18, 2004; accepted October 27, 2004)

Cardiovascular Division and *Department of Radiological Technology, Osaka Police Hospital, Osaka, Japan

Mailing address: Sei Komatsu, MD, PhD, Cardiovascular Division, Osaka Police Hospital, 10-31 Kitayama-cho, Tennoji-ku, Osaka 543-

0035, Japan._E-mail: donai@ muc.biglobe.ne.jp good correlation of the measurement of the CT image with the IVUS findings in a study of remodeling 26 However, there is not a comprehensive method for evaluating the gray-scale CT image. ROI method using a circle or rectangle is commonly used to calculate the CT number of the area of concern, but usually a circle or rectangle does not fit the shape of the plaque.

We developed the 'Plaque Map' system using the colorbased isometric lines method and Bird's Eye view to calculate the CT number in the image. Tissue characterization of the plaque and quantification of the area of both the plaque and vessel can be done by the 'Plaque Map'. In the present study we compared 'Plaque Map' with IVUS and angioscopy.

Table 1 Patients' Characteristics

\begin{tabular}{lc}
\hline \hline$N$ & 45 \\
Age (years) & $66 \pm 10$ \\
Gender (male\%) & 78 \\
Hypertension & $11(25 \%)$ \\
Hyperlipidemia & $36(80 \%)$ \\
Diabetes & $12(27 \%)$ \\
Smoking & $19(42 \%)$ \\
Vessels (LAD:LCX:RCA) & $32: 15: 31$ \\
\hline
\end{tabular}

$\overline{L A D \text {, left anterior descending coronary artery; } L C X \text {, left circumflex coro- }}$ nary artery; $R C A$, right coronary artery. 

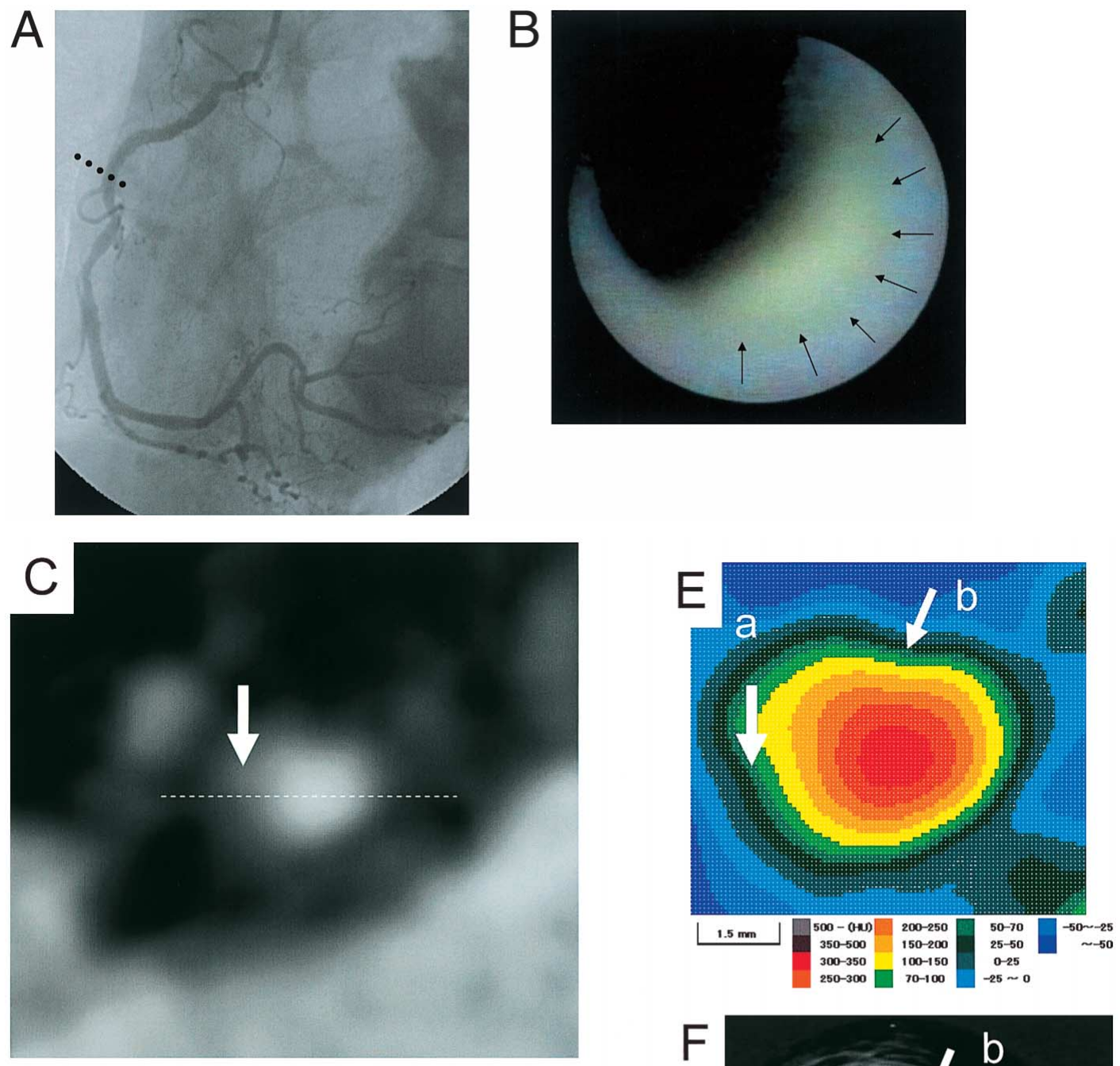

D
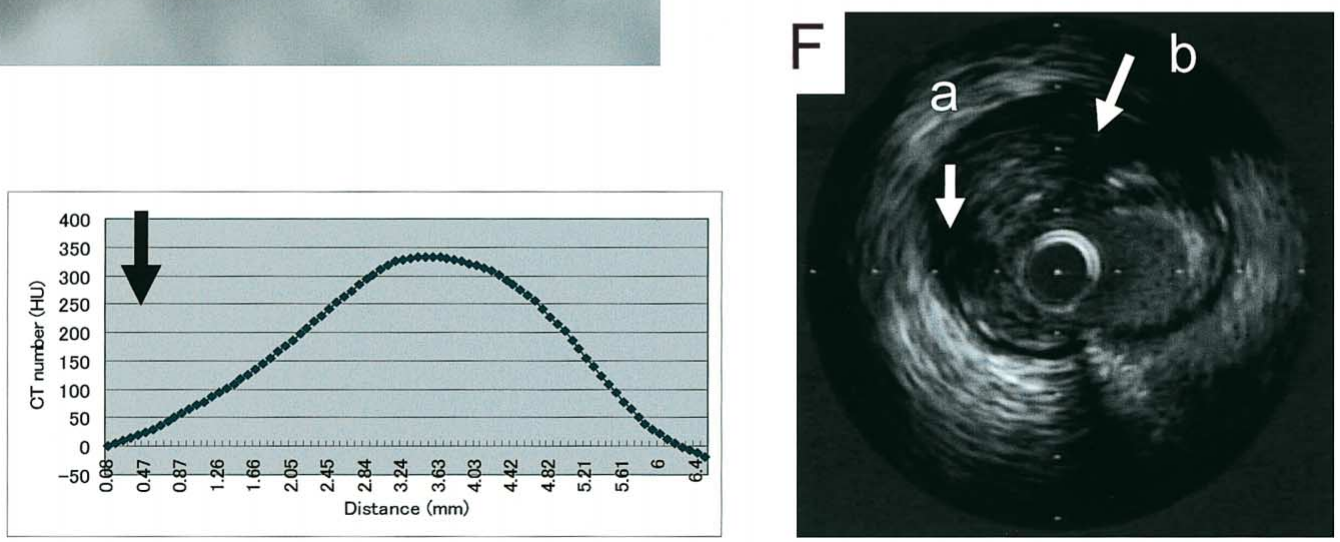

Fig 1. A 64-year old man with an inferior myocardial infarction who underwent emergency recanalization therapy with stents implanted in the right coronary artery (RCA). (A) Coronary angiography of the RCA. The cross-sections shown by the dotted line distal to stent was compared. (B) Angioscopic image of the middle of the RCA (arrows). The grade of yellow plaque was 2. (C) Axial image of the coronary plaque in (A) Arrow indicates soft plaque. (D) Profile curve from patient shown in Fig 2A. Arrow indicates plaque with a curve <100 HU. (E) 'Plaque Map' image of the middle of the RCA. a. and b. (shown as arrows) are the areas with a CT number $<100 \mathrm{HU}$, indicating soft plaque. (F) IVUS of the middle of the RCA. a. and b. are soft plaque and correspond with (E-a) and (E-b).

\section{Methods}

\section{Patients}

We studied 45 cases of ACS admitted to Osaka Police Hospital from March 2003 to February 2004. There were 24 patients with acute myocardial infarction (AMI) and 21 patients with unstable angina (UA). All patients underwent successful percutaneous coronary intervention (PCI) after admission to hospital. The study protocol was approved by the institutional ethics committee and written informed consent was obtained from all patients.

\section{Image Acquisition}

The MDCT, IVUS and angioscopy images were obtained 

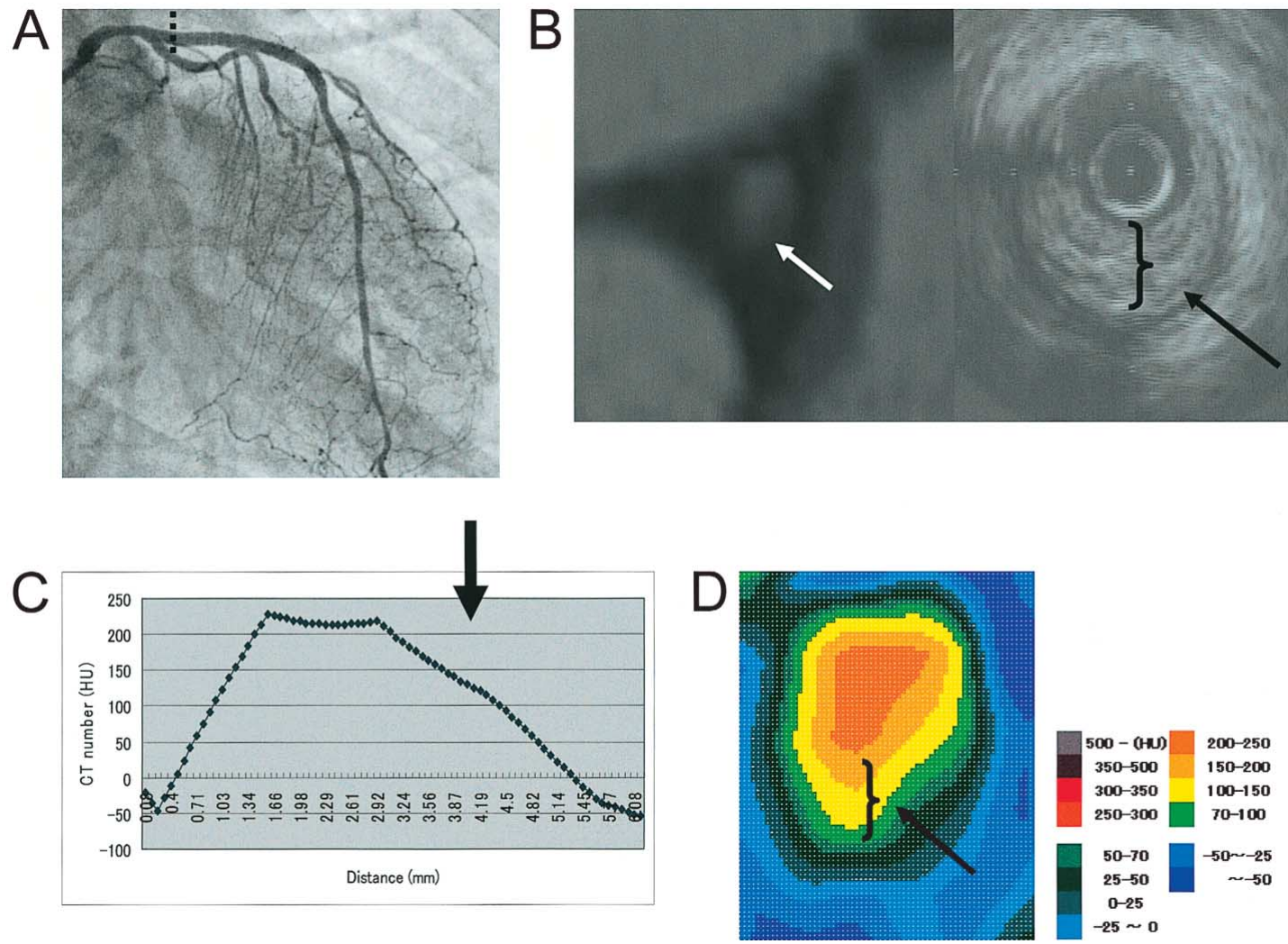

Fig 2. A 60-year old woman with an anterior myocardial infarction who underwent emergency recanalization therapy with stents implanted in the left anterior descending coronary artery (LAD). (A) Coronary angiography of the LAD showing the area of dotted line. (B, Left) Axial MDCT image proximal to the stent. (Right) IVUS image of the LAD has hard eccentric plaque (arrow). (C) Profile curve of the dotted line in Fig 3B. Intermediate plaque is indicated by the arrow. (D) 'Plaque Map' image proximal to the stent. Expansion of the region of 100-200 HU (arrow) indicates intermediate plaque.

either 3-4 weeks after the onset of AMI or within 1 week of PCI in patients with UA.

MDCT Protocol One hour before imaging patients were given metoprolol $20 \mathrm{mg}$ po and nitroglycerin sl $10 \mathrm{~min}$ before. MDCT images were obtained using a commercially available 8-detector CT scanner Light Speed Ultra (GE Systems, USA) with ECG gating and Advantage Workstation 4.0 software. As a pre-injection for determining the circulation time for the enhanced scan, $15 \mathrm{ml}$ of contrast medium $(15 \mathrm{ml}$ at $3.5 \mathrm{ml} / \mathrm{s}$; Omnipaque 300 , Daiichi Pharmaceutical Co, Ltd, Japan) was administered via an antecubital vein. Injection of contrast medium was $80 \mathrm{ml}(3.5 \mathrm{ml} / \mathrm{s})$. Each slice was $1.25 \mathrm{~mm}$ thick. Pitch was $0.625: 1$. Rotation time was $0.5 \mathrm{~s}$ and Temporal resolution was $125 \mathrm{~ms}$. The trigger point was 70-80\% (R-R). Reconstruction used Snap Shot Burst Reconstruction (retrogating reconstruction). Cross-sectional coronary images were obtained by applying the curved multiplanar reformation technique at intervals of $5 \mathrm{~mm}$.

IVUS and Angioscopy The catheter system was the flat panel single detector X-ray system 'INNOVA 2000' (GE, USA). IVUS used a commercially available Atlantis $2.9 \mathrm{Fr}, 0.5 \mathrm{~mm} / \mathrm{s}$ auto-pullback. Cross-sectional images were taken at intervals of $5 \mathrm{~mm}$ (every $10 \mathrm{~s}$ ) and the images were analyzed by Tape Measure Ver 2.5.0c. For angioscopy, we used the MC-800E and optic fiber AS-003 (Nihon Kohden, Japan) with manual pullback ${ }^{5-9}$

\section{Slice Adjustment}

The cross-sections obtained at intervals of $5 \mathrm{~mm}$ by MDCT and IVUS were compared and the position was corrected by landmarks (ie, stent or branch). Continuous cross-sections that contained soft plaque on the MDCT images were compared with the angioscopic images of the plaque detected in the same position.

\section{'Plaque Map' System}

To construct the 'Plaque Map', a series of $0.4 \mathrm{~mm}$ thickness cross-sectional images of the coronary artery were obtained at intervals of $5 \mathrm{~mm}$ (exclusion criteria were poorly distinguishable cross-sectional images such as side branches, inside the stent etc). The DICOM format of the cross-section was converted to a numerical matrix (512x 512 ) and the text data were analyzed semi-automatically by our 2 original methods: the color-based isometric line method, which colors the relevant limit of CT numbers, and the Bird's Eye View, which shows 3 dimensional (D)contour images. The borders of the isometric lines of -50 , $-25,0,25,50,70,100,150,200,250,300,350$, and 500 Hounsfield units (HU) were drawn. We named this semiautomatic system the 'Plaque Map' system and the reconstructed color image as the 'Plaque Map'. We wrote the program using Microsoft Visual Basic 6.0 in Windows 2002 and the 3D construction used Gsharp Ver 3.0 (KGT Inc, Japan). 

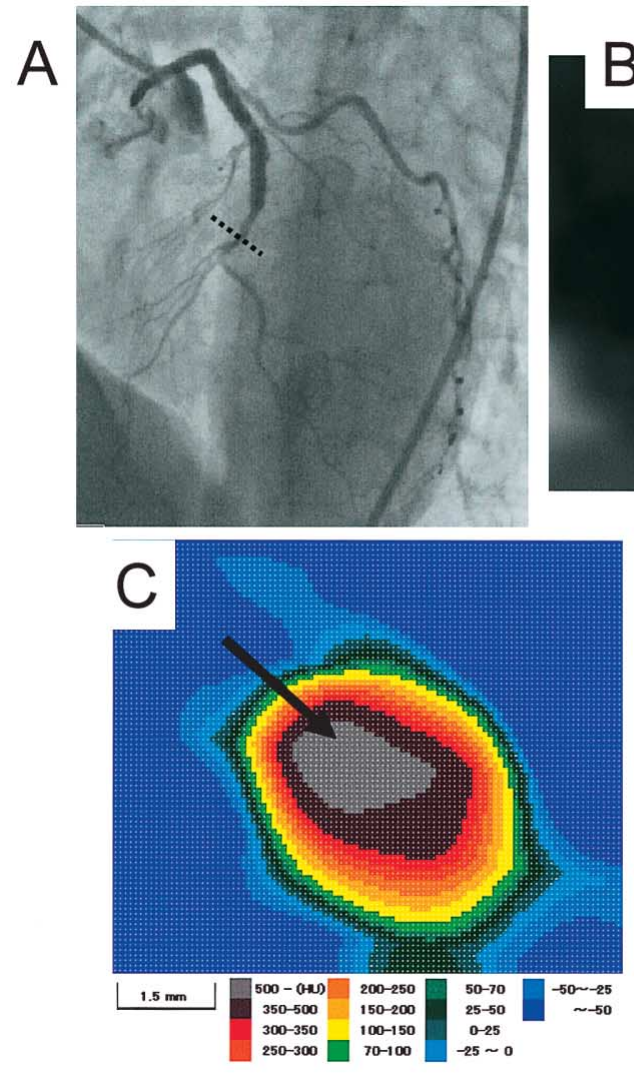
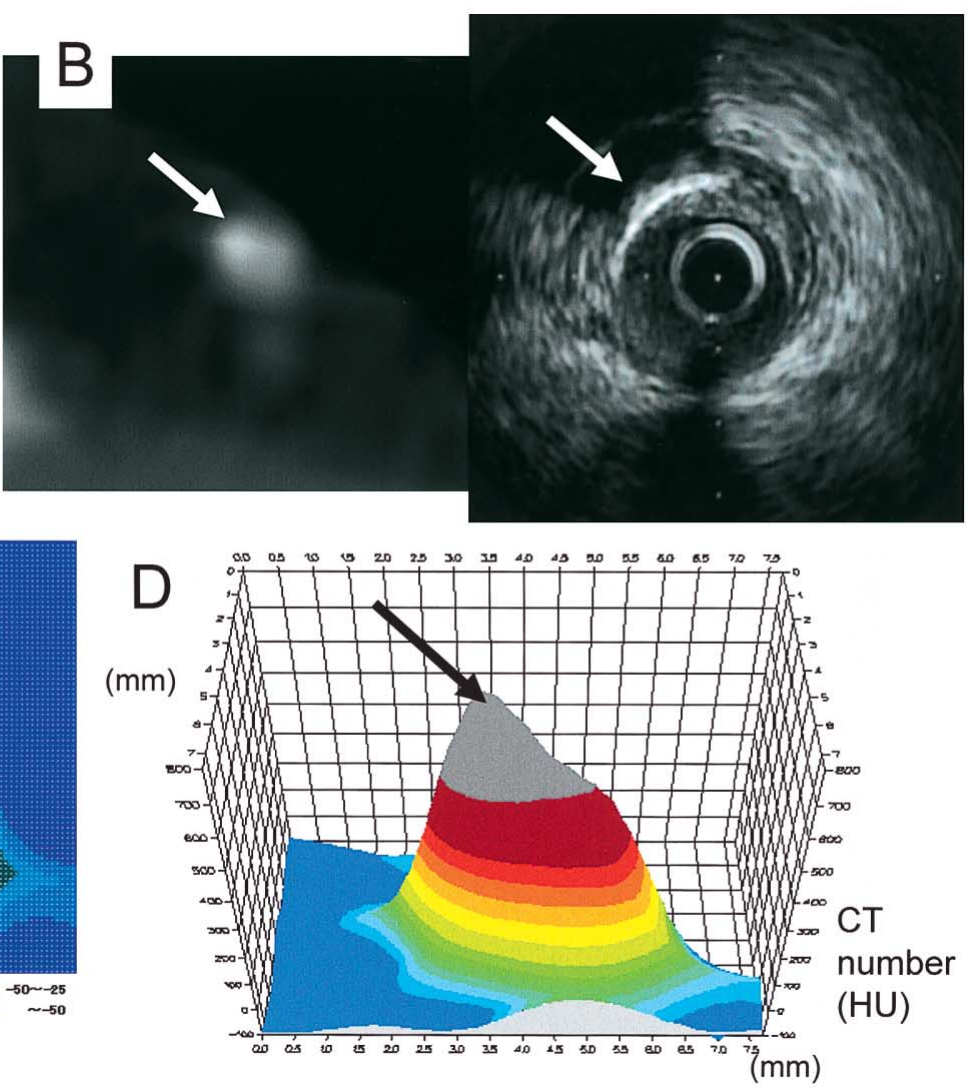

Fig 3. A 66-year old woman with an anterior myocardial infarction who underwent percutaneous coronary intervention with stents implantated in the anterior descending coronary artery. (A) Coronary angiography of the left coronary artery (LCA) showing the area of dotted line. (B, Left) Axial MDCT image distal to the stent shows high-density plaque (arrow). (Right) IVUS image of the left anterior descending coronary artery showing high echoic plaque with an acoustic shadow (arrow). (C) 2D-Plaque Map constructed by the color-based isometric line method. The arrow shows the CT number $>500$, indicating calcified plaque. (D) Bird's Eye view of the 'Plaque Map' (3D-Plaque Map). The peak CT number of this calcified plaque was $759 \mathrm{HU}$.

Table 2 Comparison of Soft, Intermediate, and Calcified Plaques by MDCT and IVUS

\begin{tabular}{lrcc}
\hline \hline & \multicolumn{3}{c}{ IVUS } \\
\cline { 2 - 4 } & Soft & Intermediate & Calcified \\
\hline MDCT-positive & 144 & 134 & 84 \\
MDCT-negative & 12 & 19 & 10 \\
Sensitivity(\%) & 92 & 87 & 89
\end{tabular}

IVUS, intravascular ultrasonography; MDCT, multi-detector row computed tomography.

\section{Definition of Plaque}

IVUS and Angioscopy The same vessels were imaged by IVUS and angioscopy. Determination of the plaque classification was based on established criteria of echogenicity ${ }^{2}$ Soft plaque was defined as more than $80 \%$ of the plaque area composed of tissue with lower echogenicity than the adventitia (arc of lesion calcium <90\%). Hard plaque was defined as more than $80 \%$ of the plaque area composed of tissue producing echoes as bright or brighter than the adventitia but without acoustic shadows (arc of lesion calcium $<90 \%$ ). Calcified plaque was defined as plaque involving bright echoes with acoustic shadowing accompanying more than $90 \%$ of the vessel wall circumference.

Yellow plaque was defined as a yellow area on the
Table 3 Comparison of Soft, Intermediate, and Calcified plaques by MDCT and Angioscopy

\begin{tabular}{lcc}
\hline \hline & \multicolumn{2}{c}{ Angioscopy (yellow plaque) } \\
\cline { 2 - 3 } & Present & Absent \\
\hline MDCT & & \\
Soft-positive & 111 & 44 \\
Soft-negative & 28 & 291 \\
\hline
\end{tabular}

Sensitivity $=80 \%$, sensitivity $=87 \%$. MDCT, multi-detector row computed tomography.

lumen surface, which may have a smooth or irregular surface with or without protrusion into the lumen. Evaluation of the IVUS and angioscopic images was performed by 2 independent observers who had no knowledge of the clinical data.

'Plaque Map' Soft, intermediate, and calcified plaque were defined as having a profile curve $<50 \mathrm{HU}, 50-150 \mathrm{HU}$, and $>500 \mathrm{HU}$ (other than for a stent), respectively, based on previous reports ${ }^{20,25,28}$

\section{Counting Plaque by 'Plaque Map' and Comparison With} IVUS and Angioscopy

We counted the number of short-axis sections with soft, intermediate, and hard plaque and compared the plaque characteristics of 'Plaque Map' with those of IVUS and 
angioscopy.

\section{Results}

\section{Detection of Coronary Plaque}

The number of vessels analyzed by MDCT, IVUS, and angioscopy is shown as Table 1 . The number of short-axis sections was 662 of 78 vessels ( $13 \pm 11$ sections/vessel). The 'Plaque Map' system detected soft plaque in 144 slices. Fig 1 shows a slice from 'Plaque Map', the IVUS image of soft plaque and the angioscopic image of yellow plaque. IVUS detected 156 cases of soft plaque, 153 of intermediate plaque, and 94 of calcified plaque. Angioscopy detected 139 yellow plaques (3.0 per person). MDCT failed to detect 12 cases of soft plaque that were detected by IVUS only. In the comparison of MDCT with IVUS, the sensitivity was $92 \%$. The sensitivity and specificity of detecting soft plaque by 'Plaque Map' were $80 \%$ and $87 \%$, respectively, when compared with yellow plaque detection by angioscopy.

We defined a gentle slope of $50-150 \mathrm{HU}$ as intermediate plaque (Fig 2). In 'Plaque Map', the slope $<100 \mathrm{HU}$ was steep, but the slope between 100 and 200 was gentle. MDCT detected intermediate plaque in 134 slices, but failed to detect 19 cases detected by IVUS, so the sensitivity was $87 \%$.

MDCT detected calcified plaque (Fig 3) in 84 slices, but did not detect 10 cases detected by IVUS, giving a sensitivity of $89 \%$.

\section{Discussion}

We have developed a semi-automatic plaque imaging and analysis system called 'Plaque Map', which provides colored 2D- (Figs 1E, 2D, 3C) or 3D- (Fig 3D) reconstructed images based on the CT number of the vessel. The system enabled us to detect and characterize coronary artery plaque.

A previous report suggested tissue characterization can be decided by CT number, ${ }^{20}$ but it is not easy when using gray-scale CT images. ROI measurement may calculate the mean CT number and standard deviation, but it does not fit the plaque. When the coronary artery is filled with contrast media, the CT number decreases linearly to the border of the vessel. Therefore, plaque characterization should be interpreted not only by the sole CT number but by pattern recognition of the distribution of the CT number of the whole vessel. The isometric lines of 'Plaque Map' enabled demonstration of the distribution of the CT number and pattern recognition.

Although IVUS is an excellent method for defining plaque from the histological and immunohistochemical findings ${ }^{14}$ and angioscopy can detect yellow plaque that may be lipid-rich with a thin fibrous cap 5,6 both techniques have limitations. IVUS is invasive $e^{10,13-15}$ and geometric distortion can degrade the cross-sectional images of the vessel wall ${ }^{29}$ Also, the amount of calcified plaque may be underestimated by IVUS $3^{30}$ Angioscopy only provides the information about the inside of the vessel and lead to discrepancy about the amount of fibrous cap fully covering the soft plaque. MDCT has poor spatial resolution for detecting the fibrous cap, but can assess all coronary arteries and side branches with a diameter of $1.5 \mathrm{~mm}$ or more for the presence of stenoses exceeding $50 \%$ diameter reduction? 26
In the present study there was a high sensitivity between the detection rate by MDCT vs IVUS and MDCT vs angioscopy. Using the 'Plaque Map' system for MDCT, we can noninvasively detect and characterize plaque with higher reliability than the present techniques $3^{31}$ However, to detect vulnerable patients, 3,4 we should integrate all the findings from MDCT, IVUS and angioscopy. In the acute stage of ACS, soft plaque and thrombus are hard to distinguish on IVUS and although none of the present subjects were in the acute phase of ACS, the CT number of soft plaque and thrombus may not have been distinguished.

\section{Study Limitations}

All patients underwent MDCT after PCI, not before, because MDCT requires approximately $100 \mathrm{ml}$ of iv contrast media. This limitation of contrast media needs to be overcome. We did not examine inside the stent because of the halation of the metal and thus the cause of ACS was not examined.

\section{Conclusion}

Our plaque imaging and analysis method, 'Plaque Map', can detect plaque characteristics as well as IVUS and angioscopy in patients with ACS.

\section{Acknowledgments}

We thank Jyouta Oyabu, Taku Sakai, and Yoshiyuki Ebihara for technical assistance and Nagisa Yoshioka, Atsuko Hayashida, Naoko Ikemachi, Yoshiko Kurata, and Midori Komatsu for their assistance with the manuscript.

\section{References}

1. Fuster V, Badimon L, Badimon JJ, Chesebro JH. The pathogenesis of coronary artery disease and the acute coronary syndromes. $N$ Engl J Med 1992; 326: 242-250.

2. Davies MJ, Thomas AC. Plaque fissuring: The cause of acute myocardial infarction, sudden ischaemic death, and crescendo angina. $\mathrm{Br}$ Heart J 1985; 53: 363-373.

3. Naghavi M, Libby P, Falk E, Casscells SW, Litovsky S, Rumberger J, et al. From vulnerable plaque to vulnerable patient: A call for new definitions and risk assessment strategies. Part I. Circulation 2003; 108: $1664-1672$.

4. Naghavi M, Libby P, Falk E, Casscells SW, Litovsky S, Rumberger $\mathrm{J}$, et al. From vulnerable plaque to vulnerable patient: A call for new definitions and risk assessment strategies. Part II. Circulation 2003; 108: $1772-1778$.

5. Asakura M, Ueda Y, Yamaguchi O, Adachi T, Hirayama A, Hori M, et al. Extensive development of vulnerable plaques as a pan-coronary process in patients with myocardial infarction: An angioscopic study. J Am Coll Cardiol 2001; 37: 1284-1288.

6. Kodama K, Asakura M, Ueda Y, Yamaguchi O, Hirayama A. The role of plaque rupture in the development of acute coronary syndrome evaluated by the coronary angioscope. Intern Med 2000; 39: 333 335.

7. Ueda Y, Hirayama A, Kodama K. Plaque characterization and atherosclerosis evaluation by coronary angioscopy. Herz 2003; 28: 501 504.

8. Ueda Y, Asakura M, Hirayama A, Adachi T, Kodama K. Angioscopy of culprit lesions. Cardiologia 1997; 42: 827-832.

9. Ueda Y, Ohtani T, Shimizu M, Hirayama A, Kodama K. Assessment of plaque vulnerability by angioscopic classification of plaque color. Am Heart J 2004; 2: 333-335.

10. Hodgson J, Reddy KG, Suneja R, Nair RN, Lesnefsky EJ, Sheehan HM. Intracoronary ultrasound imaging: Correlation of plaque morphology with angiography, clinical syndrome and procedural results in patients undergoing coronary angioplasty. $\mathrm{J} \mathrm{Am} \mathrm{Coll} \mathrm{Cardiol}$ 1993; 21: 35-44.

11. Moriuchi M, Saito S, Takaiwa Y, Honye J, Fukui T, Horiuchi K, et al. Assessment of plaque rupture by intravascular ultrasound. Heart Vessels 1997; Suppl 12: $178-181$.

12. Nissen S. Coronary angiography and intravascular ultrasound. Am J 
Cardiol 2001; 16: 15A-20A.

13. Nair A, Kuban BD, Tuzcu EM, Schoenhagen P, Nissen SE, Vince DG. Coronary plaque classification with intravascular ultrasound radiofrequency data analysis. Circulation 2002; 106: 2200-2206.

14. Okimoto T, Imazu M, Hayashi Y, Fujiwara H, Ueda H, Kohno N. Atherosclerotic plaque characterization by quantitative analysis using intravascular ultrasound: Correlation with histological and immunohistochemical findings. Circ J 2002; 66: 173-177.

15. Yamagishi M, Hosokawa H, Saito S, Kanemitsu S, Chino M, Koyanagi S, et al. Coronary disease morphology and distribution determined by quantitative angiography and intravascular ultrasound: Re-evaluation in a Cooperative Multicenter Intravascular Ultrasound Study (COMIUS). Circ J 2002; 66: 735-740.

16. Thieme T, Werniche KD, Meyer R, Brandenstein E, Habedank D, Hinz A, et al. Angioscopic evaluation of atherosclerotic plaques: Validation by histomorphologic analysis and association with stable and unstable coronary syndromes. J Am Coll Cardiol 1996; 28: 1-6.

17. Waxman S, Sassower MA, Mittleman MA, Zarich S, Miyamoto A, Manzo KS, et al. Angioscopic predictors of early adverse outcome after coronary angioplasty in patients with unstable and non-Q-wave myocardial infarction. Circulation 1996; 93: 2106-2113.

18. Farb A, Burke AP, Tang AL, Liang TY, Mannan P, Smialek J, et al. Coronary plaque erosion without rupture into a lipid core: A frequent cause of coronary thrombosis in sudden coronary death. Circulation 1996; 93: 1354-1363.

19. Achenbach S, Ropers D, Hoffmann U, MacNeill B, Baum U, Pohle $\mathrm{K}$, et al. Assessment of coronary remodeling in stenotic and nonstenotic coronary atherosclerotic lesions by multidetector spiral computed tomography. J Am Coll Cardiol 2004; 43: 842-847.

20. Kopp AF, Schroeder S, Baumbach A, Kuettner A, Georg C, Ohnesorge B, et al. Non-invasive characterization of coronary lesion morphology and composition by multislice CT: First result in comparison with intracoronary ultrasound. Eur Radiol 2001; 11: 16071611.

21. Sato Y, Matsumoto N, Kato M, Inoue F, Horie T, Kusama J, et al. Noninvasive assessment of coronary artery disease by multislice spiral computed tomography using a new retrospectively ECG-gated image reconstruction technique. Circ J 2003; 5: 401-405.

22. Sato Y, Kanmatsuse K, Inoue F, Horie T, Kato M, Kusama J, et al.
Noninvasive coronary artery imaging by multislice spiral computed tomography. Circ J 2003; 2: 107-111.

23. Kuettner A, Kopp AF, Schroeder S, Rieger T, Brunn J, Meisner C, et al. Diagnostic accuracy of multidetector computed tomography coronary angiography in patients with angiographically proven coronary artery disease. J Am Coll Cardiol 2004; 43: 831-839.

24. Ropers D, Baum U, Pohle K, Anders K, Ulzheimer S, Ohnesorge B, et al. Detection of coronary artery stenoses with thin-slice multidetector row spiral computed tomography and multiplanar reconstruction. Circulation 2003; 107: 664-666.

25. Schroeder S, Kopp AF, Baumbach A, Meisner C, Kuettner A, Georg $\mathrm{C}$, et al. Noninvasive detection and evaluation of atherosclerotic coronary plaques with multislice computed tomography. J Am Coll Cardiol 2001; 37: 1430-1435.

26. Achenbach S, Ropers D, Hoffmann U, MacNeill B, Baum U, Pohle $\mathrm{K}$, et al. Assessment of coronary remodeling in stenotic and nonstenotic coronary atherosclerotic lesions by multidetector spiral computed tomography. J Am Coll Cardiol 2004; 43: 842-847.

27. Schroeder S, Flohr T, Kopp AF, Meisner C, Kuettner A, Herdeg C, et al. Accuracy of density measurements within plaques located in artificial coronary arteries by X-ray multislice CT: Result of a phantom study. J Comp Assist Tomogr 2001; 25: 900-906.

28. Schoenhagen P, Tuzcu EM, Stillman AE, Moliterno DJ, Halliburton SS, Kuzmiak SA, et al. Non-invasive assessment of plaque morphology and remodeling in mildly stenotic coronary segments: Comparison of 16-slice computed tomography and intravascular ultrasound. Coron Artery Dis 2003; 14: 459-462.

29. Delachartre P, Cachard C, Finet G, Gerfault FL, Vray D. Modeling geometric artefacts in intravascular ultrasound imaging. Ultrasound Med Biol 1999; 25: 567-575.

30. Kostamaa H, Donovan J, Kasaoka S, Tobis J, Fitzpatrick L. Calcified plaque cross-sectional area in human arteries: Correlation between intravascular ultrasound and undecalcified histology. Am Heart J 1999; 137: 482-488.

31. Achenbach S, Moselewski F, Ropers D, Ferencik M, Hoffmann U, MacNeill B, et al. Detection of calcified and noncalcified coronary atherosclerotic plaque by contrast-enhances, submilliter multidetector spiral computed tomography. Circulation 2004; 109: 14-17. 